Retracted Article: On 2nd January 2018, the Editorial Board of Himalayan Physics agreed to retract this article because it is a duplication of an article published in Himalayan PhysicsVol.1 2010 pp.1-3 (DOI: http://dx.doi.org/10.3126/hj.vli0.5162).

The Himalayan Physics Vol. 6 \& 7, April 2017 (1-4)

ISSN 2542-2545

\title{
Temperature and Mass Profile of Far-infrared Skeleton Nebula
}

\author{
Binil Aryal \\ Central Department of Physics, Tribhuvan University, Kathmandu, Nepal
}

Email: aryalbinil@gmail.com

\begin{abstract}
Flux density variation of a newly discovered isolated far-infrared interstellar nebula $\left(R . A .=08^{h} 27^{m}\right.$, Dec. $=$ $\left.+25^{\circ} 54^{\prime}(J 2000)\right)$ is studied at $100 \mu \mathrm{m}$ and $60 \mu \mathrm{m}$ Infrared Astronomical Survey (IRAS) maps. The nebula contains two bright condensations ("nuclei") and several prominent filaments. The dust color temperature is estimated throughout the nebular region by dividing the $100 \mu \mathrm{m}$ map by the $60 \mu \mathrm{m}$ map and comparing the resulting flux I $(100 \mu \mathrm{m}) / \mathrm{I}(60$ values at each map location with the values given for dust grain models by Dwek (1986). It is found that the dust col temperature of the southern nucleus is $34 \pm 4 \mathrm{~K}$ and the temperature of its northern counterpart $32 \pm 4 \mathrm{~K}$. The eastern filamentary structures are slightly cooler $(20 \pm 2 \mathrm{~K})$ than the western filamentary structures $(26 \pm 3 \mathrm{~K})$. Hence, the locations of the maximum emission i.e. the nuclei of the nebula are warmer than their filaments. It is interestingly noticed that there are no hints of star formation in the nebula, suggesting interaction with ambient matter or the presence of unknown external radiation. Possible explanations of the results will be explained.

Keywords: Interstellar medium, Far infrared astronomy, Dust color, Fem peratine, IRAS-survey, Dust mass.
\end{abstract}

\section{INTRODUCTION}

Plasmas, a kind of cosmic soup comprised of molecules, atoms, electrons and ions, comprise 99 percent of the known universe. The three other known states of matter - solids, liquids and gases - make up a mere 1 percent. Plasmas are most commonly found in interstellar space, where residual hydrogen is ionized by radiation, and in stars w generating efficiencies earthbound scientists have long desired to emulate. In stars, for hydrogen nuclei to fuse into heavier nuclei, they must be fast enough to overcome a mutual electric repulsion when the hydrogen ionizes into plasma. Very hot plasmas overcome these limitations when their constituent particles collide with one another, acquiring sufficient energy to fuse, releasing enormous amount of energy. Such reactions are at the literal heart of the sun's core and for the next four or so billion years, a ready and abundant source of solar heat. If the same kind of forces that e kept the sun's nuclear furnace burning thus far could somehow be effieiently replicated terrestrially, power would be abundant and inexpensive, even in the world's remotest regions. Unlike nuclear fission, fusion poses little significant environmental threat. However, despite researchers' best efforts; technical problems have so far derailed efforts to produce practical fusion-based power. In the present we studied the variation of flux density and calculated the dust color temperature and the mass of the skeleton nebula discovered in the year 2006 by Aryal \& Weinberger.

\section{OBSERVATION}

Fig. 1 shows $3^{\circ} \times 3^{\circ} \mathrm{IRAS} 100 \mu \mathrm{m}$ image centered at R.A. $=$ $08^{\mathrm{h}} 27^{\mathrm{m}} 05$, Dec. $=+25^{\circ} 53^{\prime} 59^{\prime \prime}$ (J2000), i.e. at $l=197.79^{\circ}, b$ 31.58 . A remarkable A-shaped nebula with a skeletonlike morphology with a size of $2.5^{\circ} \mathrm{can}$ be seen. The name of this nebula as "Skeleton Nebula" (hereafter SkN) is coined by Aryal \& Weinberger (2006). In the optical, very faint and diffuse emission is visible on a Digital Sky Survey (DSS) image. The nebula belongs to the 'Category II cloud' of Odenwald (1988). Odenwald carried out optical and far-IR studies on 14 high Galactic latitude clouds with comet-like or filamentary appearances. Category II clouds, appearing as faint, diffuse reflection nebulae of nearly constant optical surface brightness but showing a clumpy internal structure, comprise the objects G64-26, G208-28, G225-66, G228-27, G239-15 as well as the Draco cloud, G90+38. Odenwald (1988) estimates that category II clouds have relatively low masses in the range $3-40 \mathrm{M}_{\text {sun }}$ and surface brightness typical for clouds illuminated by the general interstellar radiation field.

The Skeleton Nebula's two bright nuclei are located at R.A. $=08^{\mathrm{h}} 28^{\mathrm{m}} 30^{\text {s }}$, Dec. $=+25^{\circ} 43^{\prime} 58^{\prime \prime}(\mathrm{J} 2000)$ and R.A. $=$ $08^{\mathrm{h}} 27^{\mathrm{m}} 06^{\mathrm{s}}$, Dec. $=+26^{\circ} 20^{\prime} 10^{\prime \prime}(\mathrm{J} 2000)$, respectively. The angular separation between the nuclei is approximately 39 arc minute. At $100 \mu \mathrm{m}$ (Fig. 1a), the northern nucleus $\left(\mathrm{N}_{1}\right)$ displays 3 bright, short $\left(0.25^{\circ}\right)$, " arms" apparently originating in its brightest, that is central, region. One extends towards 
the north-east, the second one towards the south-west, and the third is perpendicular, extending towards the south-east. Both eastern and western arms further sharply (by $\sim 90^{\circ}$ ) bend southwards. Together with the above perpendicular arm all these extensions look like an upside down small letter omega.

The southern nucleus $\left(\mathrm{N}_{2}\right)$ bears some resemblance to a scaled up version of $\mathrm{N}_{1}$ and its arms (Fig. 1). The longest arm (in the west) extends from north to south. The apparent size of this filament is $\sim 80 \mathrm{arcmin}$. It should be noted that all arms, i.e. all filamentary structures associated with both nuclei are approximately parallel (within $\pm 15^{\circ}$ ) to each other and extend towards south or south-east. Hence, the remarkable structure of the Skeleton Nebula indicates that some external force is influencing its shape. The source of this force obviously must be located in the north-west of the nebula.

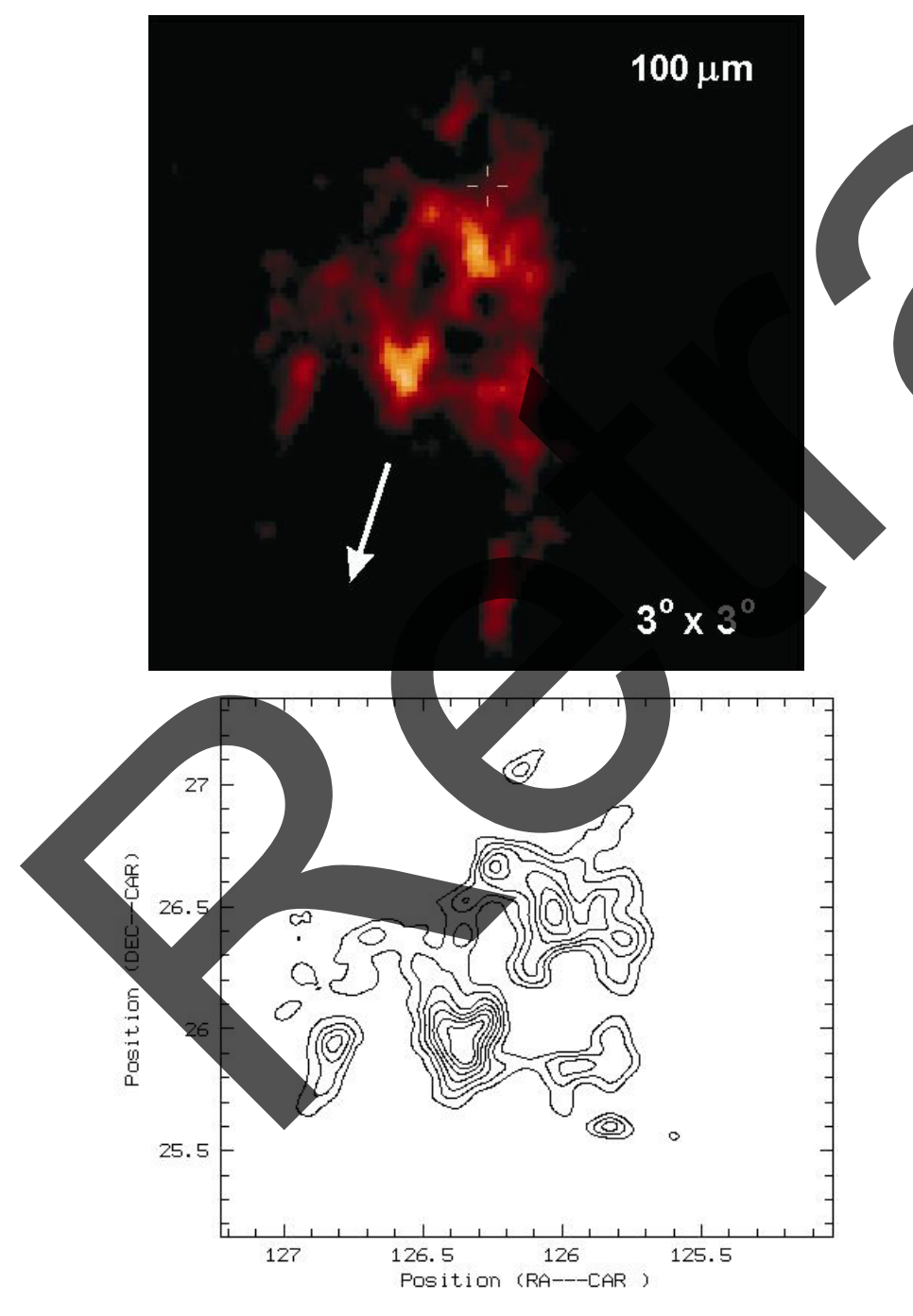

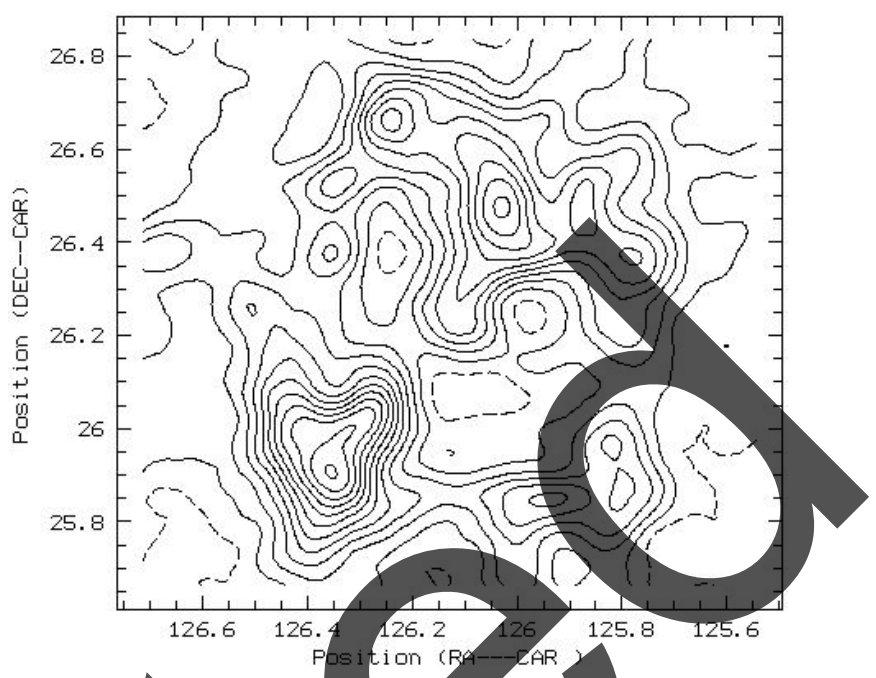

Figure 1. (a) $3^{\circ} \times 3^{\circ} \backslash \mathrm{V} A \mathrm{~S} 100 \mathrm{~mm}$ image centered at R.A. = $08^{h} 27^{m} 05^{s}$, Dec. $=+25^{\circ} 53^{\prime} 5$ 9" (J2000), i.e. at $l=197.79^{\circ}, b=$ $+31.58^{\circ}$. The arrot represents the proper motion of the pulsar. (b,c) contour maps of the field shown.

In Fig. 1b,c one notices that i) both nuclei are connected to the filamentary structures mentioned above, ii) these filamentary structures deviate towards south, iii) these structures are approximately parallel to each other, iv) there is a bridge-like connection between both nuclei, v) the vestern filamentary structures are much more extended than the eastern ones, and vi) knots can be seen in the western filament and in the northern region. There is a rapid decrease of the nebula's surface brightness towards shorter infrared wavelengths as is evident by comparing the nebula at 100 $\mu \mathrm{m}, 60 \mu \mathrm{m}, 25 \mu \mathrm{m}$ and $12 \mu \mathrm{m}$. All traces of the filamentary structures as well as both nuclei have vanished at $25 \mu \mathrm{m}$, whereas a very faint remnant of $\mathrm{N}_{2}$ remains visible at $12 \mu \mathrm{m}$. We have obtained far-IR surface brightness measurements at each of the four passbands for the nebular region using IRAS images from Groningen IRAS server (SRON, Groningen). This lack of emission at $12 \mu \mathrm{m}$ and $25 \mu \mathrm{m}$ suggests that the dust population may be relatively devoid of a small grain component as compared to the cirrus material (Puget et al. 1985), or perhaps that the small dust grain are not being adequately excited by the ambient radiation field (Mebold et al. 1985). This can be seen in Fig. 2a. The color correction depends on the shape of the intrinsic energy distribution and on the details of the wavelength response of the system. Corrections for a number of input energy distributions are given in Table 6, Chapter 6 of the 'IRAS Explanatory Supplement' (1985). The ratio of flux densities 
before the colour correction corresponds to $240 \mathrm{~K}$ of intrinsic temperature. The correction factor corresponding to this temperature as given in Table 6 is used for the color correction.
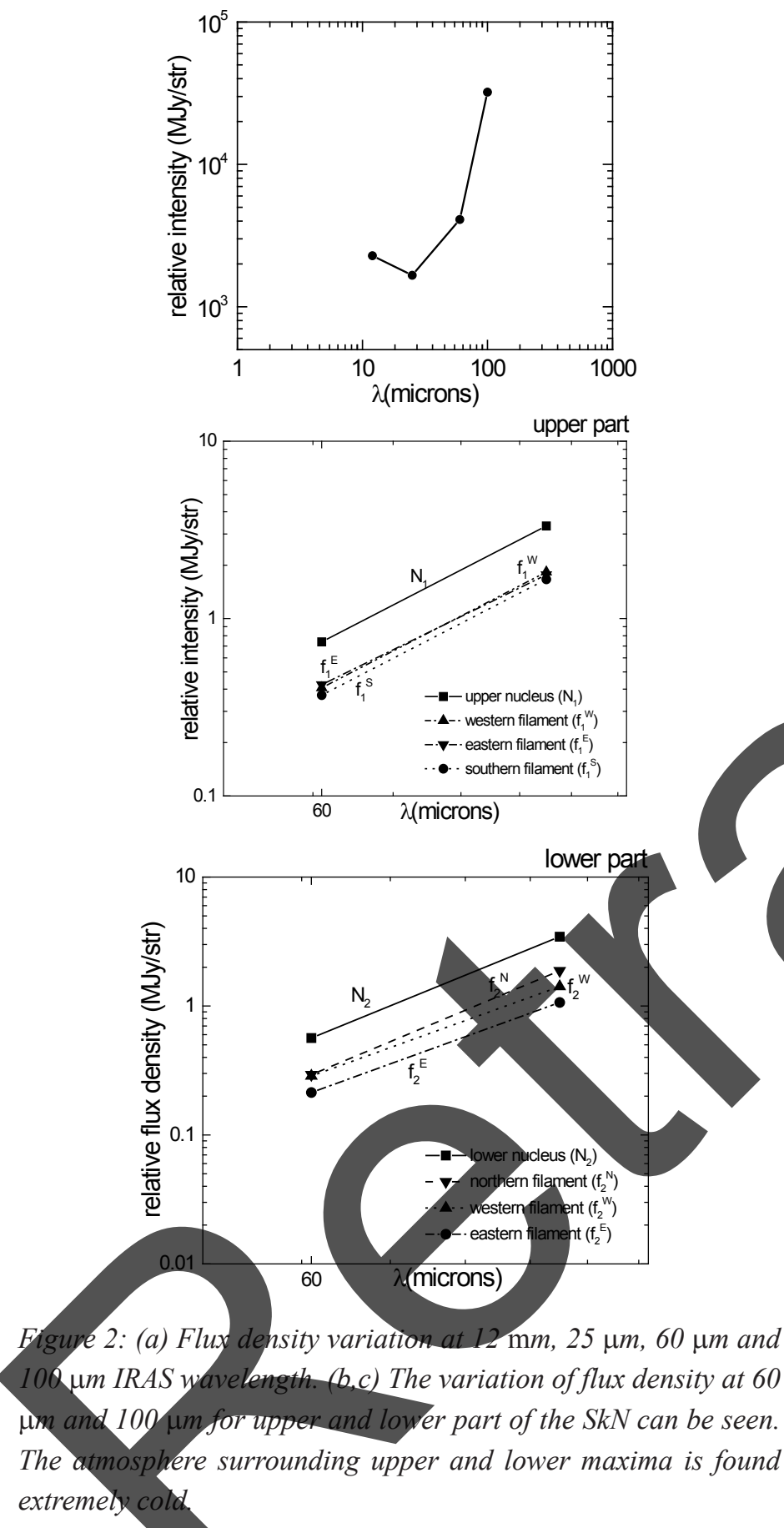

The strength of the stellar radiation field near the Draco cloud is estimated to be about 10 times lowers than in the Galactic plane (Mebold et al. 1985). $\}$ The Draco cloud is at $38^{\circ}$ latitude, at slightly higher latitude than the $\operatorname{SkN}\left(32^{\circ}\right)$. We estimate that the stellar radiation field for the $\mathrm{SkN}$ is also weaker ( $\sim 10-12$ times) with respect to a position in the
Galactic plane not only because of its high latitude but also due to the smaller number of stars in this region due to the longitude of $l=198^{\circ}$.

We used SkyView (http://skyview.gsfc.nasa.gov/) in order to search for counterparts of the Skeleton Nebula at $\mathrm{H \alpha}$ using the all-sky H $\alpha$ map, a composite of the Kirginia Tech Spectral line Survey (VTSS) in the north and the Southern H-Alpha Sky Survey Atlas (SHASSA) in the south, and in X-rays (ROSAT All Sky Survey (RASS3), RXTE/PCA All Sky Slew Survey (XSS)), as well as at radio wavelengths (Sydney University Molonglo Sky Survey (SUMS8), the VLA FIRST Survey, and the NRAO VLA Sky Survey (NVSS)), butno emission could be detected in any of these.

\section{TEMPERATURE, DISTANCE, AND MASS ESTIMATE}

Using the paper by Henning et ah. (1990) we have determined the dust color temperature $\left(\mathrm{T}_{d}\right)$ of the nebula. The average dust color temperature in the region was determined to be $27 \pm 4 \mathrm{~K}$. We also determined the dust temperature distribution throughout the nebular region by dividing the $100 \mu \mathrm{m}$ map by the $60 \mu \mathrm{m}$ map and comparing the resulting $\mathrm{I}(100 \mu \mathrm{m}) /$ $(60 \mu \mathrm{m})$ values at each map location with the values given for dust grain models by Mittaz (1999). We found that the dust color temperature of the southern nucleus is $34 \pm 4 \mathrm{~K}$ and the temperature of its northern counterpart $32 \pm 4 \mathrm{~K}$. The eastern filamentary structures are slightly cooler $(20 \pm 2 \mathrm{~K})$ than the western filamentary structures $(26 \pm 3 \mathrm{~K})$. Hence, the locations of the maximum emission i.e. the nuclei of the $\mathrm{SkN}$ are warmer than their filaments. This suggests the presence of cold plasma in the filamentary structure of the nebula.

The distance of the nebula is unknown. In our case, the star count method (Mebold et al. 1985) does not help because of insufficient extinction and too few stars in the region. The nebula also has no obviously embedded stars, which would be helpful for this purpose. Following Odenwald (1988), a distance of $200 \mathrm{pc}$ will be assumed for the moment. The maximum physical size of the nebula at this distance then is $7.6 \mathrm{pc}$, if it lies in the sky plane, and the separation between the northern $\left(\mathrm{N}_{1}\right)$ and southern $\left(\mathrm{N}_{2}\right)$ nucleus is about $2 \mathrm{pc}$.

We estimated the total mass of the SkN using the dust mass relation by Hildebrand (1983). Based on fluxes of 3.22 times $10^{4} \mathrm{MJy} / \mathrm{str}$ at $100 \mu \mathrm{m}$, and assuming a gas-to-dust mass ratio of $150, D=0.2 \mathrm{kpc}$, and $\mathrm{T}_{\mathrm{d}}=27 \pm 4 \mathrm{~K}$, the total mass 
is $3.5 \pm 2.3 \mathrm{M}_{\text {sun }}$. This value is at the lower end of the mass range of Odenwald's (1988) cometary clouds of category II $\left(3-40 \mathrm{M}_{\text {sun }}\right)$.

\section{CONCLUSION}

It is found that the dust color temperature near upper and lower maxima of the Skeleton Nebula is extremely low. It seems that the presence of a pulsar at the head of the nebula is pumping the charge particle magnetically for the existence of cold plasma.

\section{REFERENCES}

1. Aryal B. \& Weinberger R. 2006, A\&A, 365, 214

2. Henning, Th., Pfau, W., \& Altenhoff W.J. 1990, A\&A, 227,542

3. Hildebrand, R. H. 1983, Q. Jl. R. astr. Soc., 24, 267
4. IRAS Catalogs and Atlases, Explanatory Supplement 1985, ed. C. A. Beichman, G. Kulkarni Mason, K.O. et al. 2000, MNRAS, 311, 456

5. Mauron, N. et al. 2004, A\&A, 418, 77

6. Mebold, U. et al. 1985, A\&A, 151, 427

7. Melatos, A. \& Melrose, D.B. 1996, MNRAS, 279, 1168

8. Mittaz, J.P.D. et al. 1999, MNRAS, 308, 233

9. Napiwotzki, R., Green, P.J., \& Saffer, R.A. 1999, ApJ, 517,399

10. Odenwald, S.F. 1988, ApJ, 325, 320

11. Odenwald, S.F. \& Rickard, L.J 1987, ApJ, 318, 702

12. Spitzer, L. 1962, Physics of Fully Ionized Gases (NY; Interscience)

13. Weiland, J. et a1. 1986, ApJ, 306,

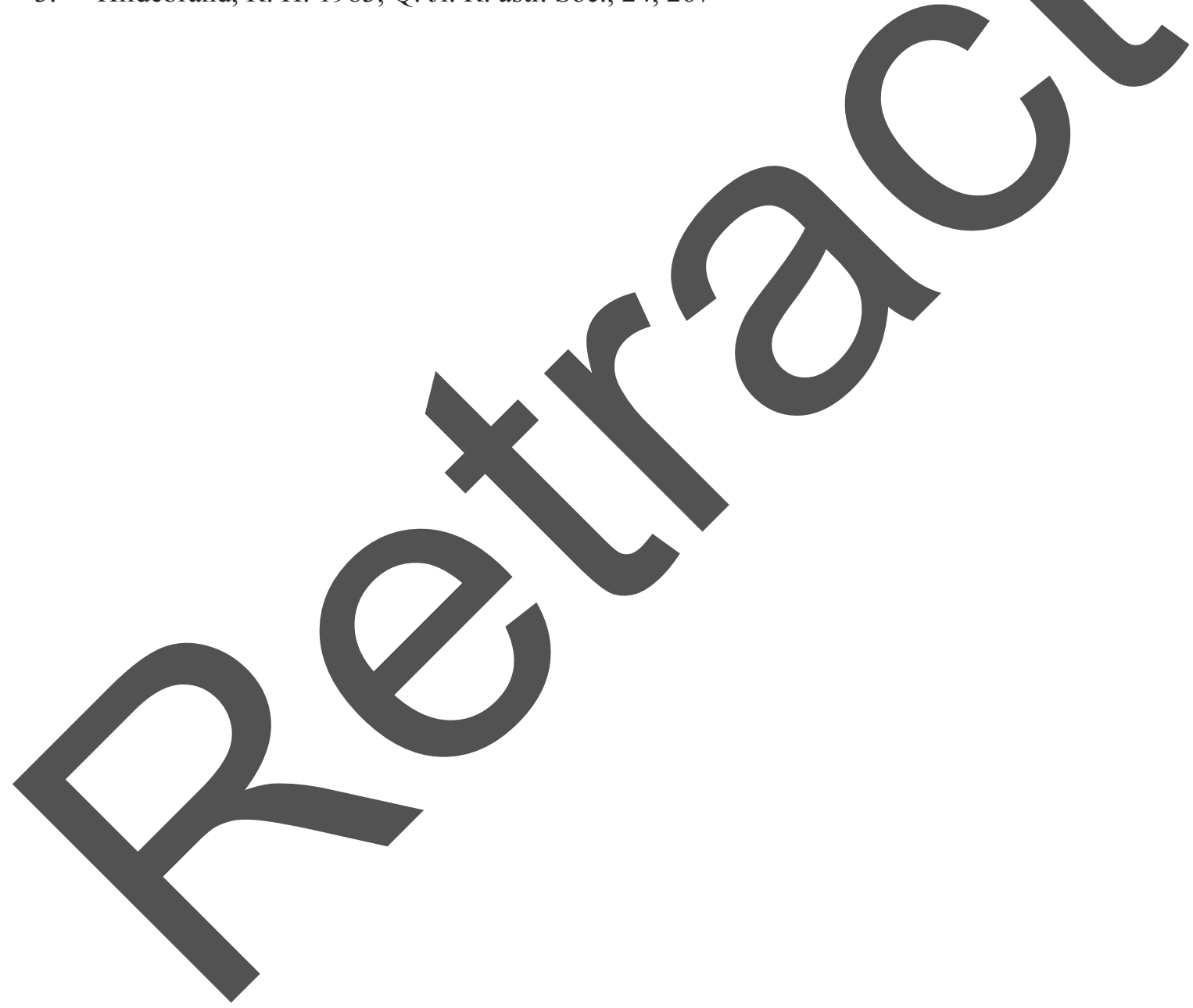

